\title{
A Study of Three Galaxy Types, Galaxy Formation, and Warm Dark Matter
}

\author{
Bruce Hoeneisen \\ Universidad San Francisco de Quito, Quito, Ecuador \\ Email: bhoeneisen@usfq.edu.ec
}

How to cite this paper: Hoeneisen, B. (2021) A Study of Three Galaxy Types, Galaxy Formation, and Warm Dark Matter. International Journal of Astronomy and Astrophysics, 11, 489-508. https://doi.org/10.4236/ijaa.2021.114026

Received: September 25, 2021 Accepted: November 20, 2021 Published: November 23, 2021

Copyright $\odot 2021$ by author(s) and Scientific Research Publishing Inc. This work is licensed under the Creative Commons Attribution International License (CC BY 4.0).

http://creativecommons.org/licenses/by/4.0/

\section{(c) (i) Open Access}

\begin{abstract}
We try to bridge the gap between the theory of linear density-velocity-gravitational perturbations in the early universe, and the relaxed galaxies we observe today. We succeed quantitatively for dark matter if dark matter is warm. The density runs of baryons and of dark matter of relaxed galaxies are well described by hydro-static equations. The evolution from initial linear perturbations to final relaxed galaxies is well described by hydro-dynamical equations. These equations necessarily include dark matter velocity dispersion. If the initial perturbation is large enough, the halo becomes self-gravitating. The adiabatic compression of the dark matter core determines the final core density, and provides a negative stabilizing feedback. The relaxed galaxy halo may form adiabatically if dark matter is warm. The galaxy halo radius continues to increase indefinitely, so has an ill-defined mass.
\end{abstract}

\section{Keywords}

Galaxy Formation, Warm Dark Matter, Adiabatic Invariant of Warm Dark Matter

\section{Introduction and Overview}

How does a particular linear density perturbation in the early universe evolve to become a relaxed galaxy that we observe today? We approach this question in (arguably) reverse chronological order. We begin with a study of relaxed elliptical galaxies with a cusp dominated by baryons. We find that the density runs $\rho_{h}(r)$ of the dark matter halo, and $\rho_{b}(r)$ of baryons, are determined (in a limited range of the radial coordinate $r$ ) by the dispersion velocities $\sqrt{\left\langle v_{r h}^{\prime 2}\right\rangle}$ of dark matter particles, and $\sqrt{\left\langle v_{r b}^{\prime 2}\right\rangle}$ of baryons, and by the radius $r_{\mathrm{eq}}$ at which $\rho_{h}\left(r_{\text {eq }}\right)=\rho_{b}\left(r_{\text {eq }}\right)$.

Next, we consider relaxed spiral galaxies with a core dominated by baryons. 
The density runs $\rho_{h}(r)$ and $\rho_{b}(r)$ are obtained by numerical integration of four coupled equations: Newton's equation, and the equation of conservation of momentum, of dark matter and of baryons. To start these numerical integrations we need four boundary conditions. These parameters are $\sqrt{\left\langle v_{r h}^{\prime 2}\right\rangle}, \sqrt{\left\langle v_{r b}^{\prime 2}\right\rangle}$, and the densities $\rho_{h}\left(r_{\min }\right)$ of dark matter, and $\rho_{b}\left(r_{\min }\right)$ of baryons, at the first measured radius $r=r_{\min }$. These four parameters cannot be obtained from the equations: they are boundary conditions that need to be obtained from observations of galaxy rotation curves, or by carrying forward the evolution of primordial density perturbations.

The purpose of the present study is to find out how nature obtains these parameters starting from a particular linear density-velocity-gravitational perturbation in the early universe. In order to simplify the problem at hand, we study spiral galaxies with a core dominated by dark matter. Then we can, to a first approximation, neglect baryons, and focus the study on the two parameters $\sqrt{\left\langle v_{r h}^{\prime 2}\right\rangle}$ and $\rho_{h}\left(r_{\min }\right)$.

Perturbations of dark matter in the early universe are described by three equations: Newton's equation, the equation of continuity, and Euler's equation. Analytic solutions in the linear approximation are well known [1]. These equations require initial conditions, i.e. the power spectrum of linear density perturbations $P(k) \tau^{2}\left(k / k_{\mathrm{fs}}\right) . P(k)$ is the power spectrum corresponding to cold dark matter [1]. If dark matter is warm, there is a cut-off factor $\tau^{2}\left(k / k_{\mathrm{fs}}\right)$ due to free-streaming, that is well approximated by

$$
\tau^{2}\left(k / k_{\mathrm{fs}}\right)=\mathrm{e}^{-k^{2} / k_{\mathrm{fs}}^{2}},
$$

(except for the case of boson dark matter with a chemical potential approaching $0^{-}$, in which case $\tau^{2}\left(k / k_{\mathrm{fs}}\right)$ develops a tail that may be searched for in future experiments) [2].

Starting with given initial perturbations, we integrate the equations numerically to study the formation of the dark matter halo of a relaxed galaxy. We can indeed obtain the parameter $\sqrt{\left\langle v_{r h}^{\prime 2}\right\rangle}$ from these integrations. However, the equations discussed so far apparently do not fix $\rho_{h}\left(r_{\text {min }}\right)$. It is sometimes assumed that various relaxation processes virialize the particles fixing $\rho_{h}\left(r_{\min }\right)$. Virialized solutions exist for any $\rho_{h}\left(r_{\min }\right)$, so $\rho_{h}\left(r_{\text {min }}\right)$ would have to be determined by the details of relaxation. So the origin of $\rho_{h}\left(r_{\min }\right)$ is a mystery. In the course of these studies, we will indeed propose a solution to the mystery, which, if true, has far reaching consequences.

We use the standard notation in cosmology as in [3].

\section{The Stationary Galactic Halo}

We consider relaxed galaxies, i.e. galaxies with no sign of recent collisions or mergers, or other extraneous features. We model the galaxy as two self-gravitating, non-relativistic, non-degenerate, gases: dark matter and baryons. The hydro-static equations that describe the density runs $\rho_{h}(r)$ of dark matter, and $\rho_{b}(r)$ of 
baryons, are Newton's equations

$$
\nabla \cdot \mathbf{g}_{h}=-4 \pi G \rho_{h}, \quad \nabla \cdot \mathbf{g}_{b}=-4 \pi G \rho_{b},
$$

and the equations of conservation of the $r$-component of momentum of particles with velocity $v_{r}>0$ (or, separately, $v_{r}<0$ ), valid for collision-less gases, or for gases with elastic collisions [4]:

$$
\nabla\left(\left\langle v_{r h}^{2}\right\rangle \rho_{h}\right)=\rho_{h}\left(\mathbf{g}+\kappa_{h} \frac{v^{2}}{r} \hat{\mathbf{e}}_{r}\right), \quad \nabla\left(\left\langle v_{r b}^{2}\right\rangle \rho_{b}\right)=\rho_{b}\left(\mathbf{g}+\kappa_{b} \frac{v^{2}}{r} \hat{\mathbf{e}}_{r}\right) .
$$

The gravitation field is $\mathbf{g}=\mathbf{g}_{b}+\mathbf{g}_{h}=-v^{2} \hat{\mathbf{e}}_{r} / r$. The velocity of rotation of test particles in circular orbits $v(r) \equiv v_{\text {tot }}(r)$ in the plane of the galaxy has contributions from dark matter and baryons: $v(r)=\sqrt{v_{h}^{2}(r)+v_{b}^{2}(r)}$. Sub-indices $h$ refer to the dark matter halo, while sub-indices $b$ refer to baryons. The constant parameters $\kappa_{h}$ and $\kappa_{b}$ are included to account (approximately) for rotation of dark matter and baryons in the disk of spiral galaxies. For spiral galaxies we have estimated $\kappa_{h} \approx 0.15$, and $\kappa_{b} \approx 0.98$ [4]. For elliptical galaxies we take $\kappa_{h} \approx 0$, and $\kappa_{b} \approx 0$. The variables $\left\langle v_{r h}^{2}\right\rangle$ and $\kappa_{h}$, and also $\left\langle v_{r b}^{2}\right\rangle$ and $\kappa_{b}$, appear in the following combinations:

$$
\left\langle v_{r h}^{\prime 2}\right\rangle \equiv \frac{\left\langle v_{r h}^{2}\right\rangle}{1-\kappa_{h}}, \quad\left\langle v_{r b}^{\prime 2}\right\rangle \equiv \frac{\left\langle v_{r b}^{2}\right\rangle}{1-\kappa_{b}} .
$$

Good fits to observed rotation curves are generally obtained assuming $\left\langle v_{r h}^{\prime 2}\right\rangle$ and $\left\langle v_{r b}^{\prime 2}\right\rangle$ are independent of the radial coordinate $r$ (in some galaxies $\left\langle v_{r h}^{\prime 2}\right\rangle$ decreases at large $r$, so the present analysis is valid up to that radius). We solve these equations in spherical coordinates, assuming spherical symmetry. These equations need four boundary conditions, e.g. $\sqrt{\left\langle v_{r h}^{\prime 2}\right\rangle}, \sqrt{\left\langle v_{r b}^{\prime 2}\right\rangle}, \rho_{h}\left(r_{\text {min }}\right)$ and $\rho_{b}\left(r_{\text {min }}\right)$. We integrate the equations numerically from the first observed radius $r_{\min }$ to the last one $r_{\max }$, in the disk of the galaxy, and vary the four boundary conditions to minimize a $\chi^{2}$ between the calculated and observed rotation curves. Note that we do not use density templates.

The observed independence of $\left\langle v_{r h}^{2}\right\rangle$ on $r$ in these relaxed galaxies implies that the particles of the gas have a 1D phase space density that follows the non-relativistic Boltzmann distribution, i.e. the number of dark matter particles in $\mathrm{d} r$ and $\mathrm{d} v_{r h}$ is proportional to [6]

$$
\exp \left[-\frac{\frac{1}{2} m_{h} v_{r h}^{2}+m_{h} \phi(r)\left(1-\kappa_{h}\right)}{k T}\right] r^{2} \mathrm{~d} r \mathrm{~d} v_{r h},
$$

where $\phi(r)=-\int_{0}^{r} g \cdot \mathrm{d} r$ is the gravitational potential. Note that from (5), $\left\langle v_{r h}^{2}\right\rangle=k T / m_{h}$, independently of $r . T / m_{h}$ is the dark matter temperature-to-mass ratio.

\subsection{Elliptical Galaxies with a Cusp Dominated by Baryons}

Numerical integrations of (2) and (3) for the elliptical galaxies M87, NGC 5846, 
and NGC 4807 are presented in Figures 1-3. The observed rotation curves are derived from measurements of the enclosed total mass $M(<r)$. The enclosed total mass, and the dark matter and baryon contributions are obtained from light profiles, velocity dispersion of stars, planetary nebulae, and globular clusters, X-ray properties from emitting hot gas, and weak and strong gravitational lensing. For large $r$, with $M_{h}(<r)$ dominating the enclosed mass, the asymptotic solutions of (2) and (3) are

$$
\rho_{h}(r)=\frac{\left\langle v_{r h}^{\prime 2}\right\rangle}{2 \pi G r^{2}}, \text { and } \rho_{b}(r) \propto r^{-2 / \alpha},
$$
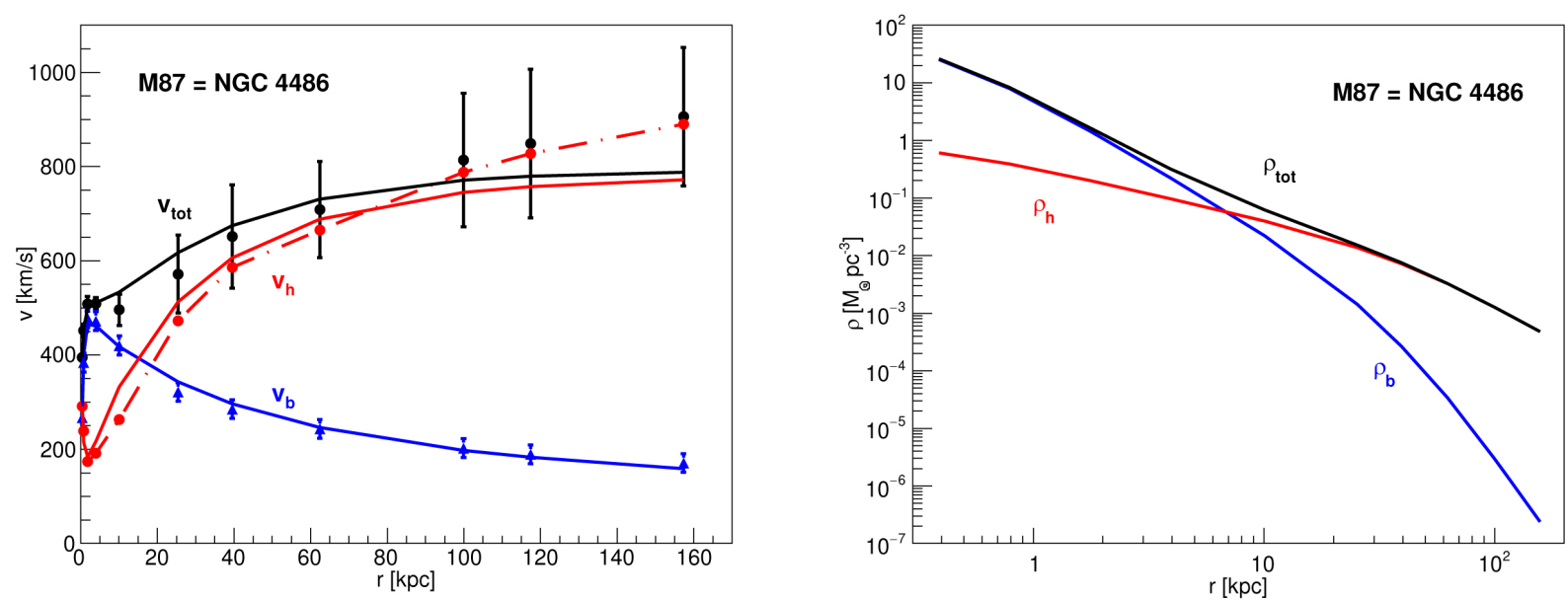

Figure 1. Velocities of circular orbits of test particles (left), and densities (right), of dark matter (sub-index $h$ ) and baryons (sub-index $b$ ), as a function of the distance $r$ from the center of the giant elliptical galaxy M87. Data is from [5]. The curves are calculated numerically, see text. The fits obtain $\sqrt{\left\langle v_{r h}^{\prime 2}\right\rangle}=533 \pm 42 \mathrm{~km} / \mathrm{s}, \quad \sqrt{\left\langle v_{r b}^{\prime 2}\right\rangle}=331 \pm 6 \mathrm{~km} / \mathrm{s}, \quad \rho_{h}\left(r_{\min }\right)=0.6 \pm 0.3 M_{\odot} / \mathrm{pc}^{3}$, and $\rho_{b}\left(r_{\min }\right)=25 \pm 3 M_{\odot} / \mathrm{pc}^{3}$ at $r_{\min }=0.39 \mathrm{kpc}$. The fitted mass-to-luminosity ratio is $\Upsilon=1.0 \pm 0.1 M_{\odot} / L_{\odot}$.
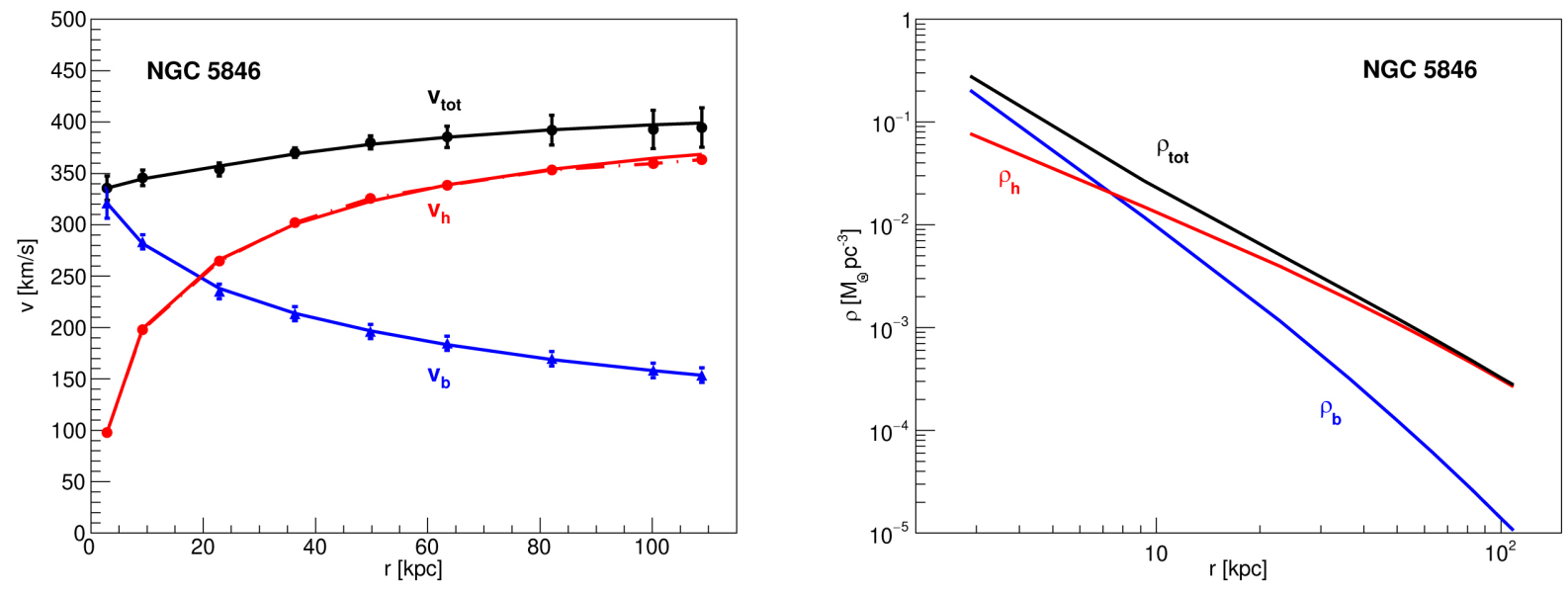

Figure 2. Velocities of circular orbits of test particles (left) and densities (right), of dark matter (sub-index $h$ ) and baryons (sub-index $b$ ), as a function of the distance $r$ from the center of the elliptical galaxy NGC 5846. Data is from [7]. The curves are calculated numerically, see text. The fits obtain $\sqrt{\left\langle v_{r h}^{\prime 2}\right\rangle}=288 \pm 11 \mathrm{~km} / \mathrm{s}, \sqrt{\left\langle v_{r b}^{\prime 2}\right\rangle}=218 \pm 4 \mathrm{~km} / \mathrm{s}, \quad \rho_{h}\left(r_{\min }\right)=0.08 \pm 0.02 M_{\odot} / \mathrm{pc}^{3}$, and $\rho_{b}\left(r_{\text {min }}\right)=0.20 \pm 0.05 M_{\odot} / \mathrm{pc}^{3}$ at $r_{\min }=2.88 \mathrm{kpc}$. The fit obtains a mass-to-light ratio $\Upsilon=0.96 \pm 0.23 M_{\odot} / L_{\odot}$. 

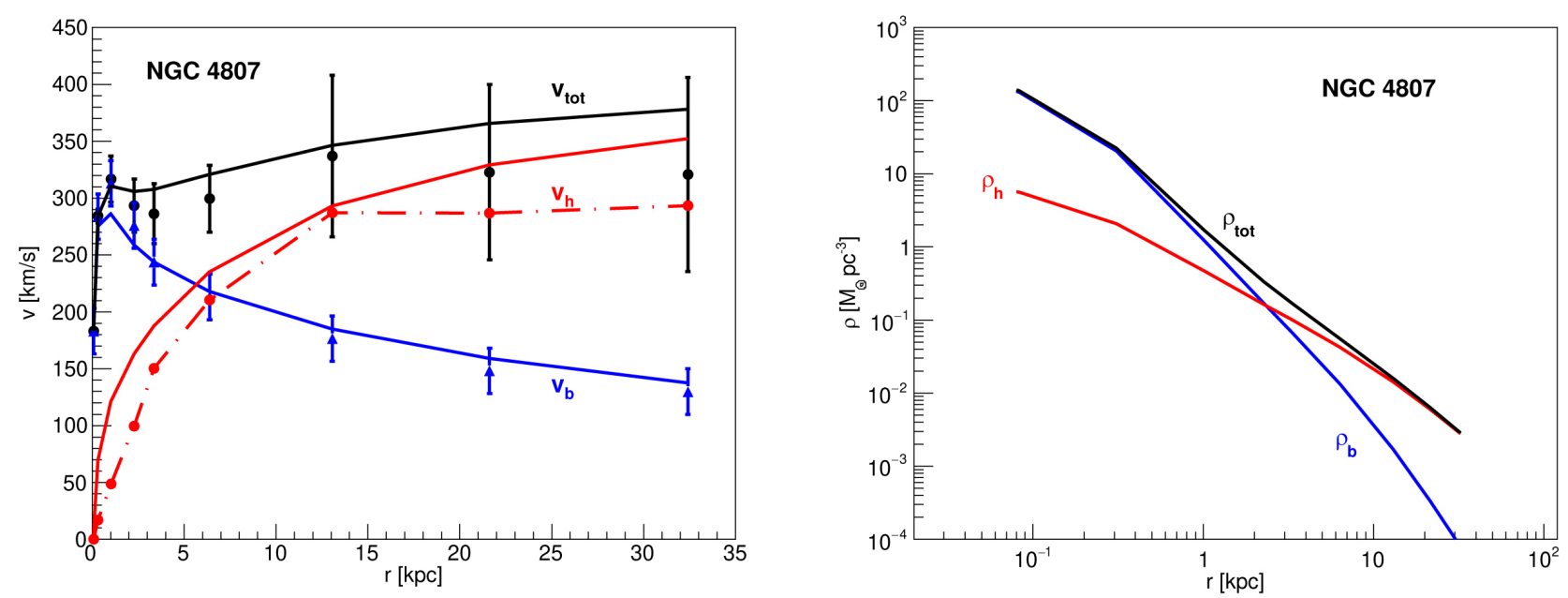

Figure 3. Velocities of circular orbits of test particles (left) and densities (right), of dark matter (sub-index $h$ ) and baryons (sub-index $b$ ), as a function of the distance $r$ from the center of elliptical galaxy NGC 4807. Data is from [8]. The curves are calculated numerically, see text. The fits obtain $\sqrt{\left\langle v_{r h}^{\prime 2}\right\rangle}=271 \pm 18 \mathrm{~km} / \mathrm{s}, \quad \sqrt{\left\langle v_{r b}^{\prime 2}\right\rangle}=197 \pm 5 \mathrm{~km} / \mathrm{s}, \quad \rho_{h}\left(r_{\min }\right)=6 \pm 4 M_{\odot} / \mathrm{pc}^{3}$, and $\rho_{b}\left(r_{\min }\right)=135 \pm 18 M_{\odot} / \mathrm{pc}^{3}$ at $r_{\min }=0.08 \mathrm{kpc}$. The mass-to-light ratio is fixed at $\Upsilon=1.0 M_{\odot} / L_{\odot}$.

where $\alpha \equiv\left\langle v_{r b}^{\prime 2}\right\rangle /\left\langle v_{r h}^{\prime 2}\right\rangle$. For small $r$, with $M_{b}(<r)$ dominating the enclosed mass, the asymptotic solutions of (2) and (3) are

$$
\rho_{b}(r)=\frac{\left\langle v_{r b}^{\prime 2}\right\rangle}{2 \pi G r^{2}} \text {, and } \rho_{h}(r) \propto r^{-2 \alpha} \text {. }
$$

Examples of galaxies that obtain good fits, with the assumption that $\sqrt{\left\langle v_{r h}^{\prime 2}\right\rangle}$ is independent of $r$, are presented in Table 1. For galaxy Mrk1216, the assumption that $\sqrt{\left\langle v_{r h}^{\prime 2}\right\rangle}$ is independent of $r$ obtains a good fit for $r<50 \mathrm{kpc}$, while $\sqrt{\left\langle v_{r h}^{\prime 2}\right\rangle}$ declines for larger $r$.

If $\alpha<2 / 3$, the baryonic mass of the galaxy is finite:

$$
M_{b} \approx \frac{2\left\langle v_{r b}^{\prime 2}\right\rangle}{G} r_{\mathrm{eq}} \frac{2-2 \alpha}{2-3 \alpha} .
$$

Replacing $M_{b}$ by $\Upsilon L$, i.e. by the mass-to-light ratio $\Upsilon$ times the absolute luminosity $L$, and taking the logarithm of (8), obtains the equation of a plane in the space $\left(\ln L, \ln \left\langle v_{r b}^{\prime 2}\right\rangle, \ln r_{\mathrm{eq}}\right)$. This plane is known in the literature as the "fundamental plane" of elliptical galaxies.

\subsection{Spiral Galaxies with a Core Dominated by Baryons}

Figure 4 presents the rotation curves and density runs of the low surface brightness spiral galaxy F571-8. At large $r$ the asymptotes are as in (6). As $r \rightarrow 0$, the densities $\rho_{h}(r)$ and $\rho_{b}(r)$ approach finite limits $\rho_{h}(r \rightarrow 0)$ and $\rho_{b}(r \rightarrow 0)$, i.e. the galaxy has a core of dark matter, and a core of baryons. Additional examples can be found in [2] and [13].

Let us understand the boundary conditions. The flat rotation velocity $v_{\text {flat }}(r)$ at large $r$ determines $\sqrt{\left\langle v_{r h}^{\prime 2}\right\rangle}=v_{\text {flat }} / \sqrt{2}$. The slope of the rotation velocity of test 

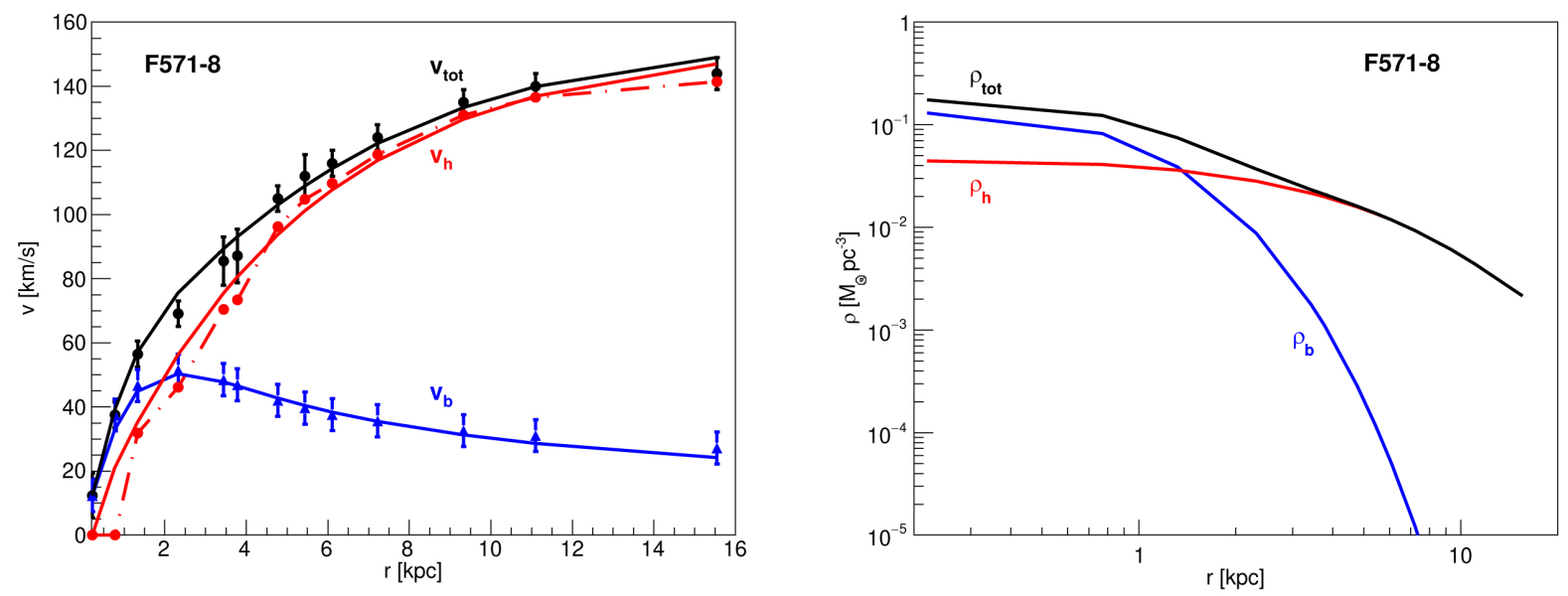

Figure 4. Velocities of circular orbits (left) and densities (right), of dark matter (sub-index $h$ ) and baryons (sub-index $b$ ), as a function of the distance $r$ from the center of the low surface brightness spiral galaxy F571-8. Data is from [12] and references therein. The curves are calculated numerically, see text. The fits obtain $\sqrt{\left\langle v_{r h}^{\prime 2}\right\rangle}=99.5 \pm 2.7 \mathrm{~km} / \mathrm{s}, \sqrt{\left\langle v_{r b}^{\prime 2}\right\rangle}=40.8 \pm 1.4 \mathrm{~km} / \mathrm{s}$, $\rho_{h}\left(r_{\min }\right)=0.044 \pm 0.004 M_{\odot} / \mathrm{pc}^{3}$, and $\rho_{b}\left(r_{\min }\right)=0.13 \pm 0.03 M_{\odot} / \mathrm{pc}^{3}$ at $r_{\min }=0.22 \mathrm{kpc}$. The disk mass-to-light ratio $\Upsilon=0.20 M_{\odot} / L_{\odot}$ is fixed. These measurements obtain the adiabatic invariant $v_{h \mathrm{rms}}(1)=\sqrt{1-\kappa_{h}} \times(1.57 \pm 0.09($ stat $)) \mathrm{km} / \mathrm{s}$, see Section 4 for the definition. $L_{3.6 \mu \mathrm{m}}=10.164 \times 10^{9} L_{\odot}$.

Table 1. Parameters $\sqrt{\left\langle v_{r h}^{\prime 2}\right\rangle}$ and $\sqrt{\left\langle v_{r b}^{\prime 2}\right\rangle}$, and their ratio $\sqrt{\alpha}$, for several elliptical galaxies with a cusp dominated by baryons. Uncertainties are statistical from the fits described in Section 2. Additional examples can be found in [9].

\begin{tabular}{ccccc}
\hline Galaxy & $\left\langle v_{r h}^{\prime 2}\right\rangle^{1 / 2}$ & $\left\langle v_{r b}^{\prime 2}\right\rangle^{1 / 2}$ & $\sqrt{\alpha}$ & $\begin{array}{c}\text { Data } \\
\text { source }\end{array}$ \\
\hline M87 & $533 \pm 42$ & $331 \pm 6$ & 0.62 & {$[5]$} \\
NGC 1407 & $291 \pm 5$ & $255 \pm 6$ & 0.88 & {$[10]$} \\
NGC 4374 & $289 \pm 4$ & $253 \pm 8$ & 0.88 & {$[11]$} \\
NGC 4807 & $271 \pm 18$ & $197 \pm 5$ & 0.73 & {$[8]$} \\
NGC 5846 & $288 \pm 11$ & $218 \pm 4$ & 0.76 & {$[7]$} \\
\hline
\end{tabular}

particles $v(r)=\sqrt{v_{h}^{2}(r)+v_{b}^{2}(r)}$, and its contribution from baryons $v_{b}(r)$, at small $r$, determine the dark matter density in the core of the galaxy: $\rho_{c} \equiv \rho_{h}(r \rightarrow 0)=3\left[v(r)^{2}-v_{b}(r)^{2}\right] /\left(4 \pi G r^{2}\right)$.

\subsection{Spiral Galaxies with a Core Dominated by Dark Matter}

Figure 5 presents the rotation curves and density runs of the spiral galaxy NGC 0024 with a core (arguably) dominated by dark matter. Additional examples can be found in [2] and [13]. First galaxies (arguably) have a core dominated by dark matter. For simplicity, in the following we will study the formation of galaxies dominated by dark matter, since then the density run $\rho_{h}(r)$ of the relaxed galaxy is described with just two parameters: the dark matter reduced velocity dispersion $\sqrt{\left\langle v_{r h}^{\prime 2}\right\rangle}$, and the dark matter core density $\rho_{c} \equiv \rho_{h}(r \rightarrow 0) \approx \rho_{h}\left(r_{\min }\right)$. 

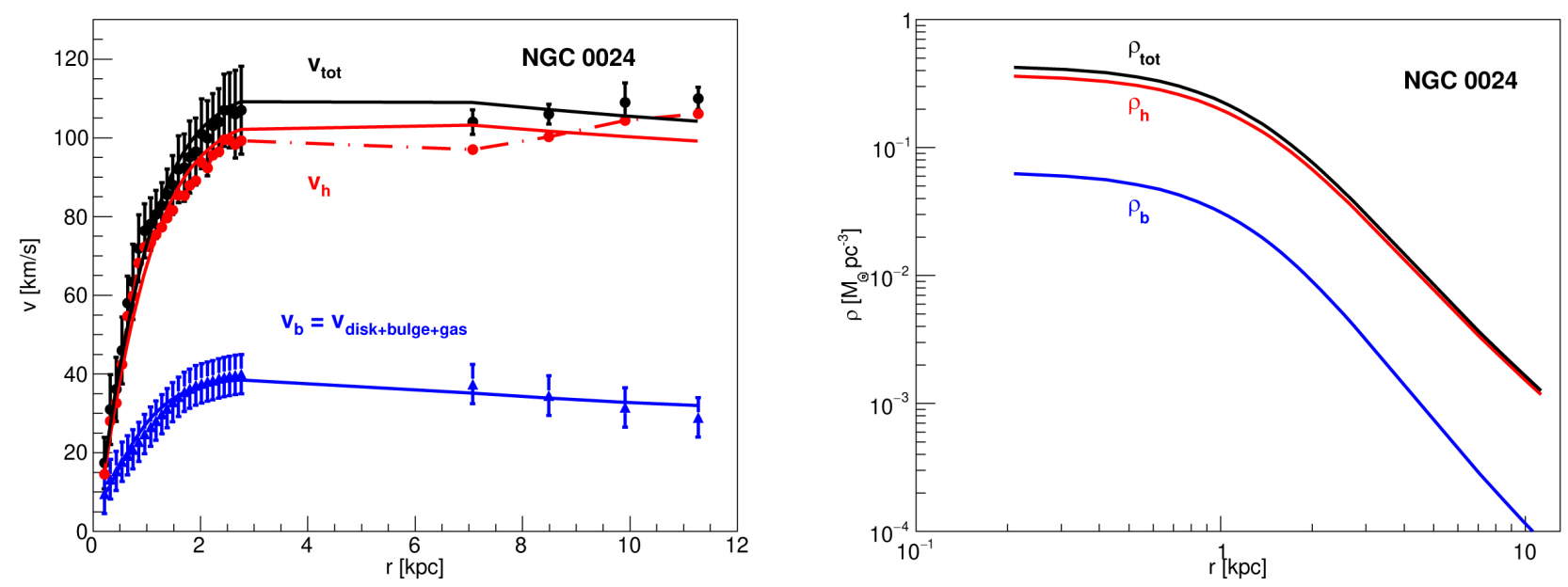

Figure 5. Velocities of circular orbits (left) and densities (right), of dark matter (sub-index $h$ ) and baryons (sub-index $b$ ), as a function of the distance $r$ from the center of the spiral galaxy NGC 0024. Data is from [12] and references therein. The mass-to-light ratio has been fixed at $\Upsilon=0.26 M_{\odot} / L_{\odot}$, but is uncertain in the approximate range 0.2 to 0.5 [12], i.e. the measurement of $\rho_{b}$ is uncertain. The curves are calculated numerically, see text. The fits obtain $\sqrt{\left\langle v_{r h}^{\prime 2}\right\rangle}=71.2 \pm 0.9 \mathrm{~km} / \mathrm{s}$, $\sqrt{\left\langle v_{r b}^{\prime 2}\right\rangle}=66.4 \pm 2.4 \mathrm{~km} / \mathrm{s}, \quad \rho_{h}\left(r_{\min }\right)=0.36 \pm 0.03 M_{\odot} / \mathrm{pc}^{3}$, and $\rho_{b}\left(r_{\min }\right)=0.062 \pm 0.007 M_{\odot} / \mathrm{pc}^{3}$ at $r_{\text {mms }}=0.21 \mathrm{kpc}$. These measurements obtain $v_{h \mathrm{rms}}(1)=\sqrt{1-\kappa_{h}} \times(0.56 \pm 0.03($ stat $)) \mathrm{km} / \mathrm{s} . \quad L_{3.6 \mu \mathrm{m}}=3.889 \times 10^{9} L_{\odot}$.

\section{Formation of the Galactic Halo}

Let us consider a universe with zero spatial curvature, dominated by non-relativistic warm dark matter. The mean density of the early universe is

$$
\bar{\rho}_{h}=\frac{1}{6 \pi G t^{2}}=\frac{\Omega_{c} \rho_{\text {crit }}}{a^{3}},
$$

where $t$ is the age of the universe, and $a(t)$ is the expansion parameter (normalized to $a=1$ today). The velocity of expansion is $v_{r}=H r$, with Hubble parameter $H=2 /(3 t)$. If dark matter is warm, the root-mean-square of the $r$-component of the dark matter particles dispersion velocity (with respect to a comoving observer) is

$$
\sqrt{\left\langle v_{r h}^{2}\right\rangle} \equiv \frac{v_{h r \mathrm{~ms}}(a)}{\sqrt{3}}=\frac{v_{h r \mathrm{~ms}}(1)}{\sqrt{3} a} .
$$

This velocity dispersion scales as $\propto 1 / a(t)$, so

$$
\frac{\sqrt{\left\langle v_{r h}^{2}\right\rangle}}{\bar{\rho}_{h}^{1 / 3}} \equiv \frac{v_{h r m s}(1)}{\sqrt{3}\left(\Omega_{c} \rho_{\text {crit }}\right)^{1 / 3}}
$$

is an adiabatic invariant. Note that the velocity $\mathbf{v}$ of a free particle (with respect to a comoving observer momentarily at the position of the particle) scales as $\mathbf{v} \propto a^{-1}$ due to the expansion of the universe.

For simplicity, we consider only dark matter. The formation of the galactic halo can be illustrated by integrating numerically Newton's equation

$$
\nabla \cdot \mathbf{g}=-4 \pi G \rho_{h},
$$


the continuity equation

$$
\frac{\partial \rho_{h}}{\partial t}=-\nabla \cdot \mathbf{j}=-\nabla \cdot\left(\mathbf{v}_{h} \rho_{h}\right)
$$

and Euler's equation

$$
\frac{\mathrm{d} \mathbf{v}_{h}}{\mathrm{~d} t}=\frac{\partial \mathbf{v}_{h}}{\partial t}+\left(\mathbf{v}_{h} \cdot \nabla\right) \mathbf{v}_{h}=\mathbf{g}-\frac{1}{\rho_{h}} \nabla\left(\left\langle v_{r h}^{\prime 2}\right\rangle \rho_{h}\right) .
$$

Newton's equation, and the last two terms in (14), obtain the steady state dark matter halo of Section 2. Again we take $\left\langle v_{r h}^{\prime 2}\right\rangle$ independent of $r$ for this steady state solution. This approximation is valid out to a radius where the rotation velocity $v(r)$ remains flat, often beyond the range of observations, and the present analysis is limited to that radius.

Linear perturbation theory is obtained for $\rho_{h}=\bar{\rho}_{h}\left(1+\delta_{h}\right)$ with $\delta_{h} \ll 1$. Equations (12), (13), and (14), with $\left\langle v_{r h}^{\prime 2}\right\rangle=0$, and in the linear approximation, admit the following solutions for the perturbations $\delta_{h}(\mathbf{r} / a), \delta \mathbf{v}_{h}(\mathbf{r} / a)$, and $\phi(\mathbf{r} / a)$ : two vector modes that decay as $\propto a^{-1}$, one scalar mode that decays as $\propto a^{-3 / 2}$, and one scalar mode that grows as $\propto a$ due to gravitational collapse, and will survive [1]. $\phi$ is the gravitational potential. $\mathbf{r}$ is the proper coordinate, and $\mathbf{r} / a$ is the co-moving coordinate. We assume dark matter is collision-less. If $\left\langle v_{r h}^{\prime 2}\right\rangle>0$, then modes with proper length

$$
\lambda<\lambda_{J} \equiv \sqrt{\left\langle v_{r h}^{\prime 2}\right\rangle \pi /\left(G \bar{\rho}_{h}\right)}
$$

are damped and collapse due to free streaming. (For collisional gases, $\sqrt{\left\langle v_{r h}^{\prime 2}\right\rangle}$ is replaced by the sound speed $c_{s}$, and $\lambda_{J}$ is the Jeans wavelength. For $\lambda<\lambda_{J}$ the solutions are sound waves. If the mean free path of the particles is comparable to $\lambda$, the sound wave is attenuated due to Silk damping [1]).

Here, to understand the formation of the dark matter halo of a galaxy, we integrate Equations (12), (13), and (14) numerically, in proper (not co-moving) spherical coordinates, and only consider spherically symmetric solutions. For warm dark matter we supplement the preceding equations with the condition of adiabatic expansion (11) in the core of the galaxy:

$$
\sqrt{\left\langle v_{r h}^{2}\right\rangle}=\frac{v_{h r \mathrm{~ms}}(1)}{\sqrt{3}}\left(\frac{\rho_{h}\left(r_{\text {min }}, t\right)}{\Omega_{c} \rho_{\text {crit }}}\right)^{1 / 3} .
$$

This equation will be discussed in Section 4, and its importance, to fix and stabilize the galaxy core density and radius, will be illustrated by the simulations. $r_{\min }$ is the smallest $r$ in the numerical integration, and is chosen much smaller than the galaxy core radius $r_{c}$.

To understand the formation of the galactic halo we need to include velocity dispersion, i.e. the last term in (14), since it is needed to obtain agreement with the observed rotation curves of relaxed galaxies as shown in Section 2. Once we include velocity dispersion, Equations (12), (13) and (14) become incomplete since $\left\langle v_{r h}^{\prime 2}\right\rangle$ remains unspecified, and we can not, consistently, omit the adiabatic invariant constraint (16). 
The simple implementation (16) of adiabatic expansion is justified as follows. To integrate Equations (12), (13), and (14) we need to assign a value to $\left\langle v_{r h}^{\prime 2}\right\rangle$, possibly $r$ - and $t$-dependent. From numerical studies described below, we find that the solutions are insensitive to $\left\langle v_{r h}^{\prime 2}\right\rangle$ for $t$ less than turn-around, or for large $r$ (beyond the pivot point discussed below). Thus, for the problem at hand, it is sufficient to assign an $r$-independent value (16) to $\left\langle v_{r h}^{\prime 2}\right\rangle$, derived from the dark matter core density $\rho_{c}(t) \approx \rho_{h}\left(r_{\text {min }}, t\right)$, at each time step in the numerical integration.

Equations (12), (13), (14), and (16) are approximate: they are hydro-dynamical equations that treat dark matter as a continuous medium, not as a superposition of particle orbits, and do not include relaxation mechanisms to damp oscillations to attain a relaxed final state. Relaxation is beyond the scope of the present study. For a review of cosmological simulations of galaxy formation see [14].

As an example we consider a galaxy with stellar mass $M_{*}=10^{9.5} M_{\odot}$, observed at a redshift $z=8$, see Figure 4 of [15], or Figure 10 of [2]. At $z=8$, the mean density of the homogeneous universe is $\bar{\rho}_{i}=2 \times 10^{-5} M_{\odot} \mathrm{pc}^{-3}$. We estimate the halo dark matter mass to be $M_{h} \approx 10^{11} M_{\odot}$ [2] [15]. We interpret this mass $M_{h}$ as the mass of the initial linear density perturbation since it is well defined: the dimensions of this perturbation scale as the expansion parameter $a$, while its density scales as $a^{-3}$ (for $\delta_{h} \ll 1$ ), so $M_{h}$ of linear perturbations is independent of time. (As we shall see later, the mass of the relaxed halo is ill defined). We will start the simulation at $\bar{\rho}_{i}=0.03 M_{\odot} \mathrm{pc}^{-3}$, corresponding to $a=1 / 97$. The radius of the initial linear perturbation is then of order $9 \mathrm{kpc}$.

Figure 6 and Figure 7 illustrate the formation of the halo of this galaxy. In this example, the initial linear density perturbation is Gaussian, $\rho_{i}=\bar{\rho}_{i}\left[1+\delta \exp \left(-r^{2} / r_{i}^{2}\right)\right]$, with $\bar{\rho}_{i}=0.03 M_{\odot} \mathrm{pc}^{-3}, r_{i}=4.9 \mathrm{kpc}$, and $\delta=0.3$. The initial dark matter velocity is the Hubble flow, and the dark matter adiabatic invariant, in this simulation, is $v_{h \mathrm{rms}}(1)=0.67 \mathrm{~km} / \mathrm{s} \quad[13]$. We note, in Figure 6 , that the central density decreases with time, turns around, increases, and approaches the final steady-state galaxy halo with asymptotes marked with lines $\mathrm{C}$ $\mathrm{D}$ E. The increase of the core density is due to negative dark matter velocity $v_{r h}$ after turn-around shown in Figure 7. This dark matter is falling back to the core.

Let us recall that the final steady-state galaxy halo is determined by (12), (13), and (14) with $\partial / \partial t \rightarrow 0$ and $v_{r h}=0$, i.e. by Newton's Equation (12), and the momentum conservation equation

$$
\mathbf{g}=\frac{1}{\rho_{h}} \nabla\left(\left\langle v_{r h}^{\prime 2}\right\rangle \rho_{h}\right)
$$

These static equations require two boundary conditions, namely the dark matter reduced velocity dispersion $\sqrt{\left\langle v_{r h}^{\prime 2}\right\rangle}$ (taken to be independent of $r$ ), and the core density $\rho_{c} \equiv \rho_{h}(r \rightarrow 0) \approx \rho_{h}\left(r=r_{\text {min }}\right)$. Let us also recall that these two parameters where obtained, in Section 2, by comparing the observed and calculated galaxy rotation curves. 


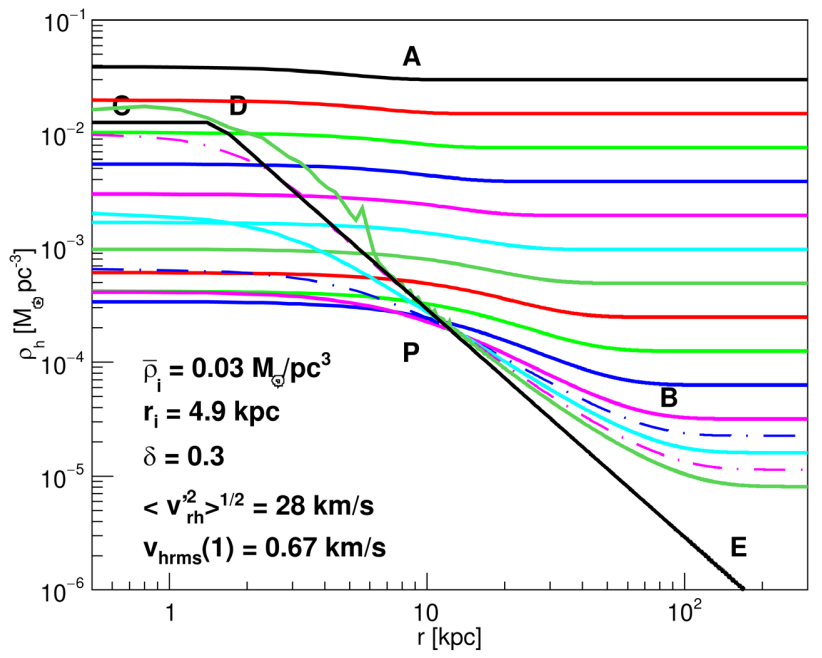

Figure 6. The dark matter density run $\rho_{h}(r)$ of a galaxy with stellar mass $M_{*}=10^{9.5} M_{\odot}$ observed at redshift $z=8$, is presented at times $t \propto \rho^{-1 / 2} \propto a^{3 / 2}$ that increase by factors 1.4086 (or $\sqrt{1.4086}$ for the dot-dashed lines). A line from A to B encloses a dark matter mass $M_{h} \approx 10^{11} M_{\odot}$. Asymptotes of the final dark matter halo $\rho_{h}(r)$ are indicated by lines C D E. The core density C is $\rho_{c}=0.0126 M_{\odot} / \mathrm{pc}^{3}$. The asymptote D E is $\rho_{h}=\left\langle v_{r h}^{\prime 2}\right\rangle /\left(2 \pi G r^{2}\right)$ with $\sqrt{\left\langle v_{r h}^{\prime 2}\right\rangle}=28 \mathrm{~km} / \mathrm{s}$, as determined by the pivot point $\mathrm{P}$. The dark matter velocity dispersion $\sqrt{\left\langle v_{r h}^{2}\right\rangle}$ in the core of the galaxy is calculated, at each time step, with (16) with $v_{h \mathrm{rms}}=0.67 \mathrm{~km} / \mathrm{s}$. We take $\kappa_{h}=0$. The last line is invalid: it is included to illustrate numerical integration breakdown, see Figure 7.

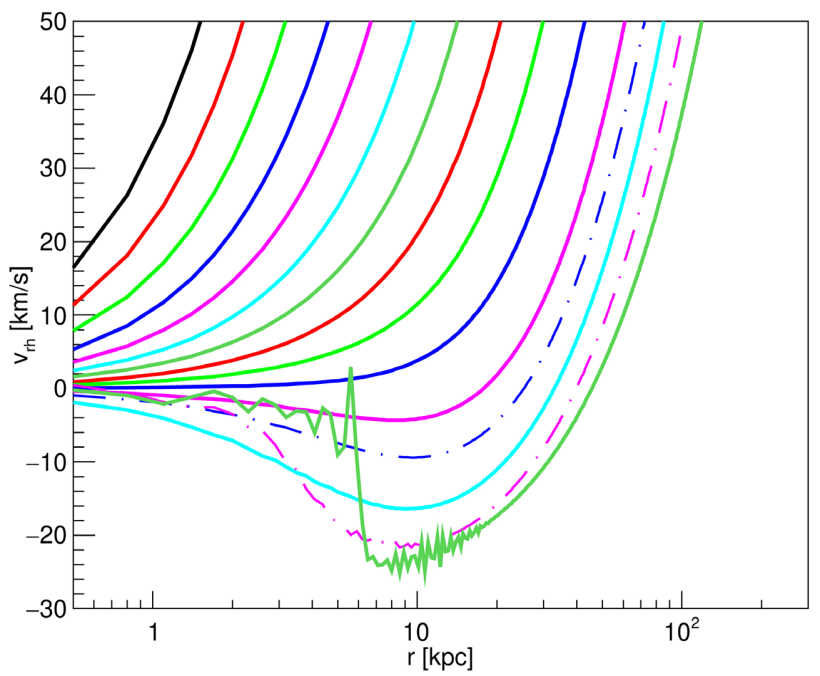

Figure 7. Shown is the radial velocity $v_{r h}(r)$ of dark matter as a function of radius $r$ at several times for the galaxy of Figure 6 . Note that adiabatic compression feedback sets $v_{r h}=0 \mathrm{~km} / \mathrm{s}$ out to a radius $r$ that increases with time $t$, up to the pivot point where the numerical integration algorithm breaks down. We expect $v_{r h}$ to reach zero out to a radius that increases past $\mathrm{P}$ at a velocity $\sqrt{3\left\langle v_{r h}^{\prime 2}\right\rangle}$, thus attaining the relaxed configuration of Section 2 out to increasing radii. This adiabatic compression feedback is negative, i.e. it tends to stabilize the core density. 
The main purpose of the present study is to determine how these two parameters, $\sqrt{\left\langle v_{r h}^{\prime 2}\right\rangle}$ and $\rho_{c}$, are obtained from the linear density-velocity-gravitational perturbations of the early universe. It turns out that the pivot point $P$ in Figure 6 fixes the final halo asymptote D E. We find that the asymptote D E depends on the initial linear density perturbation, see Figure 8 , but not on the adiabatic invariant $v_{h \mathrm{rms}}(1)$, see Figure 9 . The pivot point $\mathrm{P}$ is determined by numerical integration, starting with the initial linear perturbation, as shown in Figure 8. The asymptote $\mathrm{D} \mathrm{E}$, for $r \gg r_{c}$, is

$$
\rho_{h}(r)=\frac{\left\langle v_{r h}^{\prime 2}\right\rangle}{2 \pi G r^{2}}, \quad M_{h}(<r)=\frac{2\left\langle v_{r h}^{\prime 2}\right\rangle}{G} r,
$$

as in (6). Therefore the pivot point $\mathrm{P}$ determines the final reduced velocity dispersion $\sqrt{\left\langle v_{r h}^{\prime 2}\right\rangle}$. The galaxy core radius is defined by

$$
r_{c} \equiv\left[\frac{\left\langle v_{r h}^{\prime 2}\right\rangle}{2 \pi G \rho_{c}}\right]^{1 / 2} .
$$
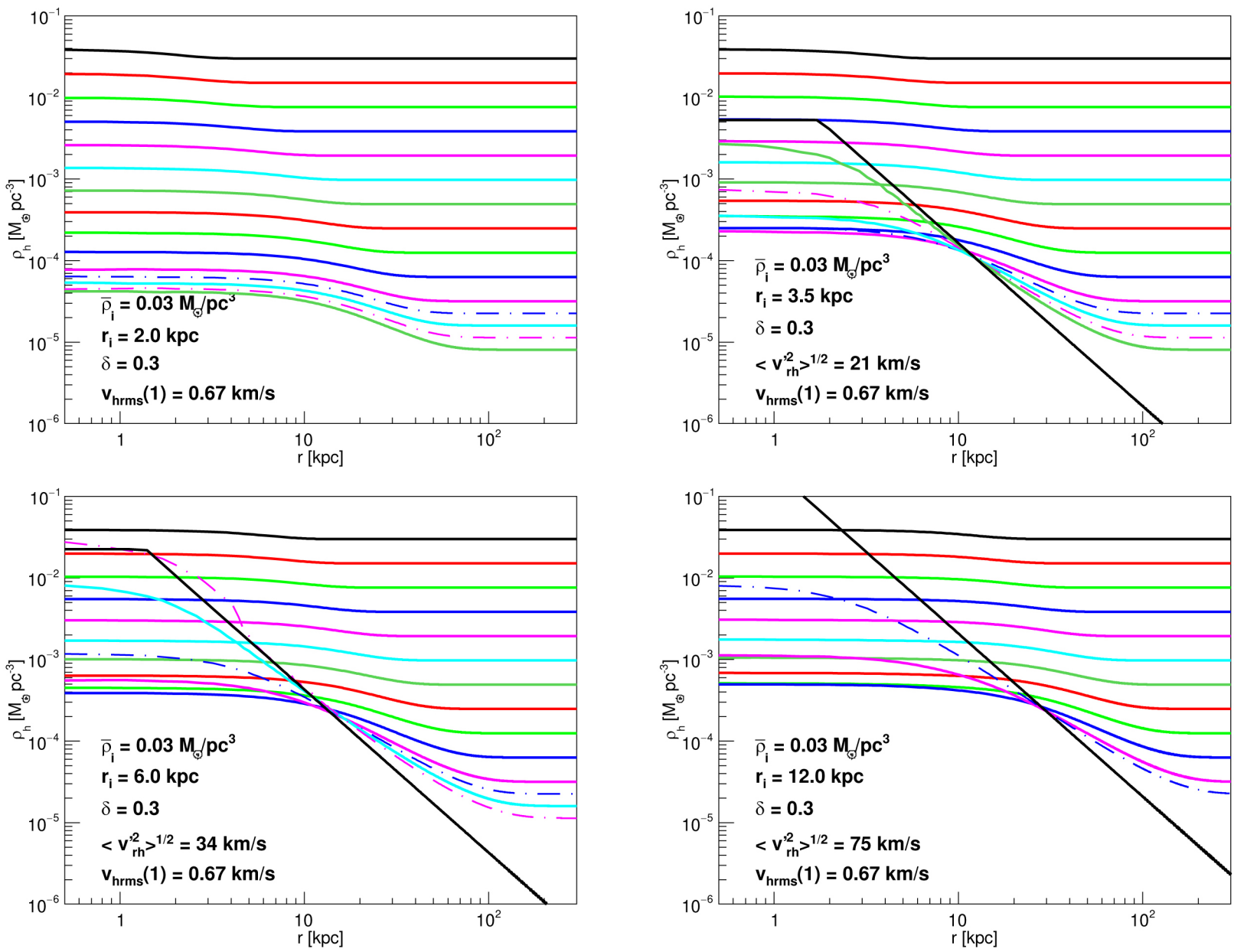

Figure 8. Same as Figure 6, except that the radius of the Gaussian perturbation is set at $r_{i}=2.0,3.5,6.0$ or $12.0 \mathrm{kpc}$. Note that the initial perturbation determines $\sqrt{\left\langle v_{r h}^{\prime 2}\right\rangle}$. 

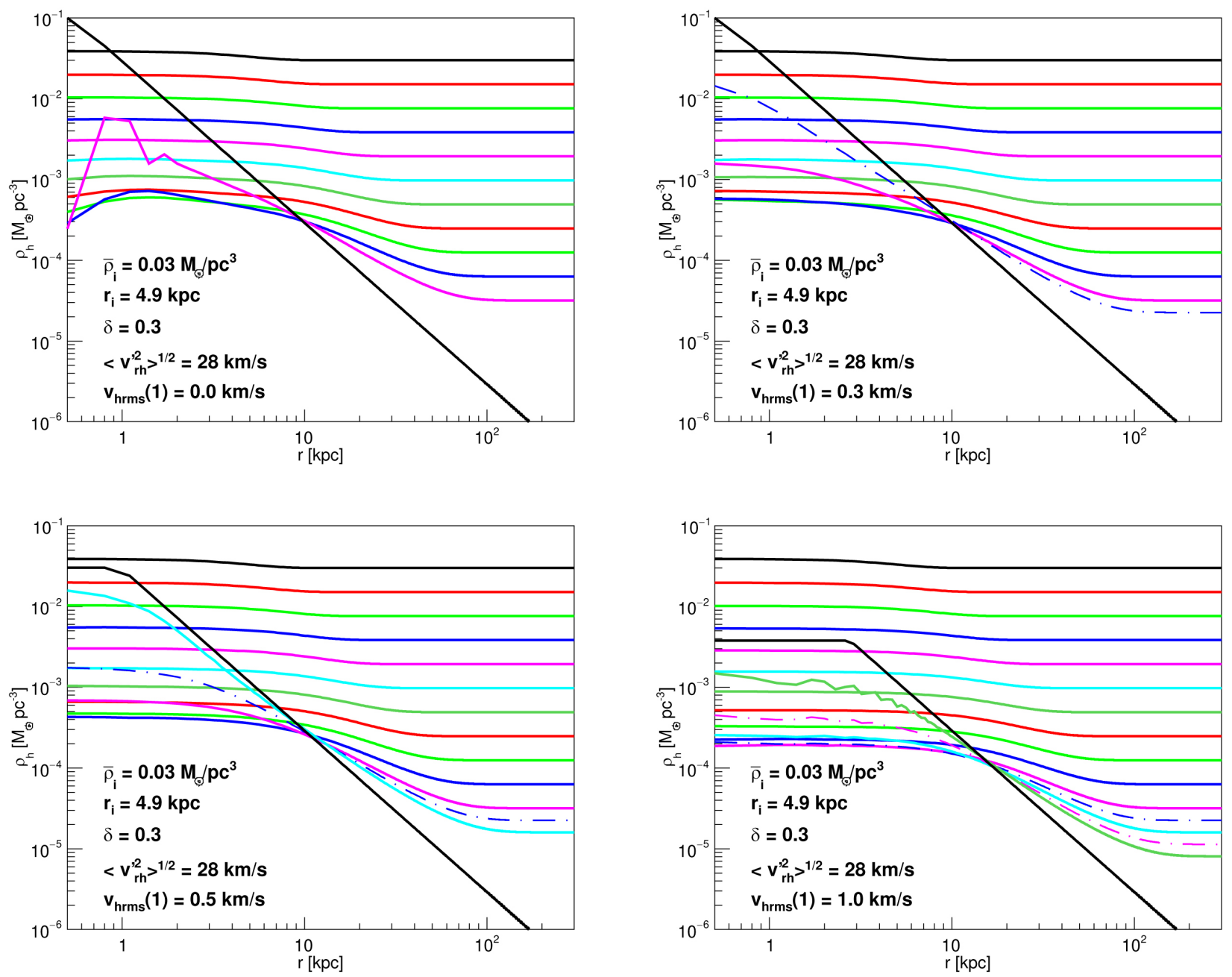

Figure 9. Same as Figure 6, except that the dark matter adiabatic invariant is set to $v_{h r m s}(1)=0.0,0.3,0.5$, or $1.0 \mathrm{~km} / \mathrm{s}$. Note that $\sqrt{\left\langle v_{r h}^{\prime 2}\right\rangle}$ of the asymptote is independent of $v_{h r m s}(1)$.

The condition to obtain a self-gravitating core is that the core radius at turn-around be less than the would be pivot point radius, so that a pivot point can form. Passing this bottle-neck, the core collapses, see Figure 6 and Figure 8.

What phenomena determines the second parameter $\rho_{c}$ ? In the cold $\Lambda \mathrm{CDM}$ scenario, the central density increases to infinity, i.e. the dark matter particles fall to $r \approx 0$, overshoot, splash-back, overshoot again, etc., and various relaxation mechanisms, and the virial theorem (assuming a well-defined halo mass $M_{h}$ ), are invoked to finally attain a relaxed galaxy halo [16]. These phenomena are not captured by Equations (12), (13), and (14), and the numerical integration breaks down at core collapse, see the first panel of Figure 8 .

In the case of warm dark matter we complement Equations (12), (13), and (14) with the equation of adiabatic expansion (16). Note, in Figure 10, that the value of $\sqrt{\left\langle v_{r h}^{\prime 2}\right\rangle}$ does not change the pivot point $\mathrm{P}$, and affects (does not affect) the halo formation for $r$ less than (greater than) $r$ of the pivot point. For this reason 

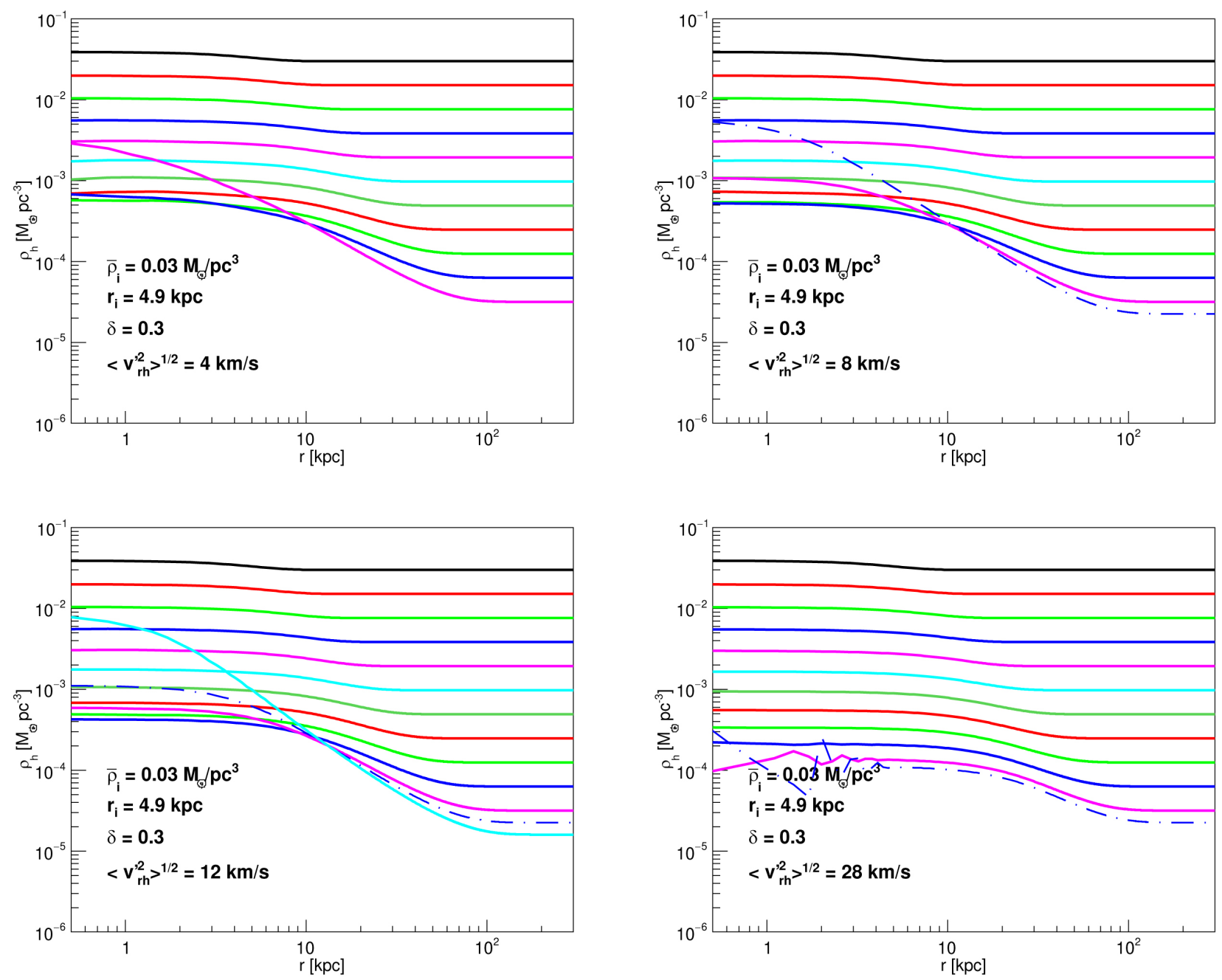

Figure 10. Same as Figure 6, except that the dark matter reduced velocity dispersion $\sqrt{\left\langle v_{r h}^{\prime 2}\right\rangle}$ is held fixed at 4, 8, 12, or $28 \mathrm{~km} / \mathrm{s}$. To keep the core $\sqrt{\left\langle v_{r h}^{\prime 2}\right\rangle}$ independent of time is un-natural, but helps understand galaxy halo formation. Note that the value of $\sqrt{\left\langle v_{r h}^{\prime 2}\right\rangle}$ does not change the pivot point $\mathrm{P}$, and affects (does not affect) the halo formation for $r$ less than (greater than) $r$ of the pivot point.

we use the core density $\rho\left(r_{\min }, t\right)$ to update $\sqrt{\left\langle v_{r h}^{\prime 2}\right\rangle}$ at each time step using (16). After core turn-around, the core density $\rho_{c}$ increases, and hence $\sqrt{\left\langle v_{r h}^{\prime 2}\right\rangle}$ increases. This is a negative feedback on the further growth of $\rho_{c}$, see Figure 10. In summary, (16) not only determines the steady-state core density (once the pivot point $\mathrm{P}$, and the final $\sqrt{\left\langle v_{r h}^{\prime 2}\right\rangle}$ are determined), but also provides a negative feedback that tends to stabilize the core density. With fixed $\left\langle v_{r h}^{\prime 2}\right\rangle$ the collapse is run-away.

Note in Figure 6 and Figure 7 that dark matter keeps falling onto the steady state galaxy halo, so the radius of this halo continues to grow. Equating the halo density (18) with the density (9) of the homogeneous universe, we obtain the constant velocity with which the halo grows: $r / t=\sqrt{3\left\langle v_{r h}^{\prime 2}\right\rangle}$. Note that the aver- 
age velocity of a dark matter particle in the halo times the age of the universe $t$, is of the order of the radius of the halo at time $t$. Note also that the halo mass keeps growing so is an ill defined concept. Let us mention that dark matter particles with orbits well within the boundary of the halo at time $t$ have completed many orbits in time $t$. So $\left\langle v_{r h}^{\prime 2}\right\rangle$ in the core and in the inner asymptotic region are connected, and attain thermal equilibrium. Finally, let us mention that, as the galaxy halo radius grows, new dark matter particles acquire orbits bound to the galaxy, populating the tail of the non-relativistic Boltzmann distribution.

As the central density $\rho_{c}$ in Figure 6 increases, so does the core temperature due to adiabatic compression. This compression brings $v_{r h}$ to its equilibrium value $v_{r h}=0$ out to a radius $r$ that increases with time, see Figure 7. Numerical integration breakdown occurs when $v_{r h}=0$ approaches the pivot point $\mathrm{P}$. The negative $v_{r h}$ beyond $\mathrm{P}$ indicates that the dark matter halo radius keeps growing. If we artificially "jump" the pivot point $\mathrm{P}$, i.e. we stop the numerical integration before $v_{r h} \approx 0$ reaches $\mathrm{P}$, and resume the integration after passing $\mathrm{P}$, we obtain the result shown in Figure 11 (left panel). In nature we do not see any significant extraordinary phenomena at $\mathrm{P}$, see, for example, the figures in Section 2. Neither do we see any instability in the integration of the static equations, see Figure 11 (right panel).

Figure 12 shows the formation of the halo of a galaxy starting from a density perturbation that has both positive and negative fluctuations. Note how the halo keeps growing past the pivot point $\mathrm{P}$.

Figure 13 shows the formation of the galactic halo starting from a top-hat density perturbation. It is interesting to note that even in this extreme case the galaxy halo forms adiabatically. Note how the transition region from high-to-low density widens. The analytic solutions inside and outside of the transition
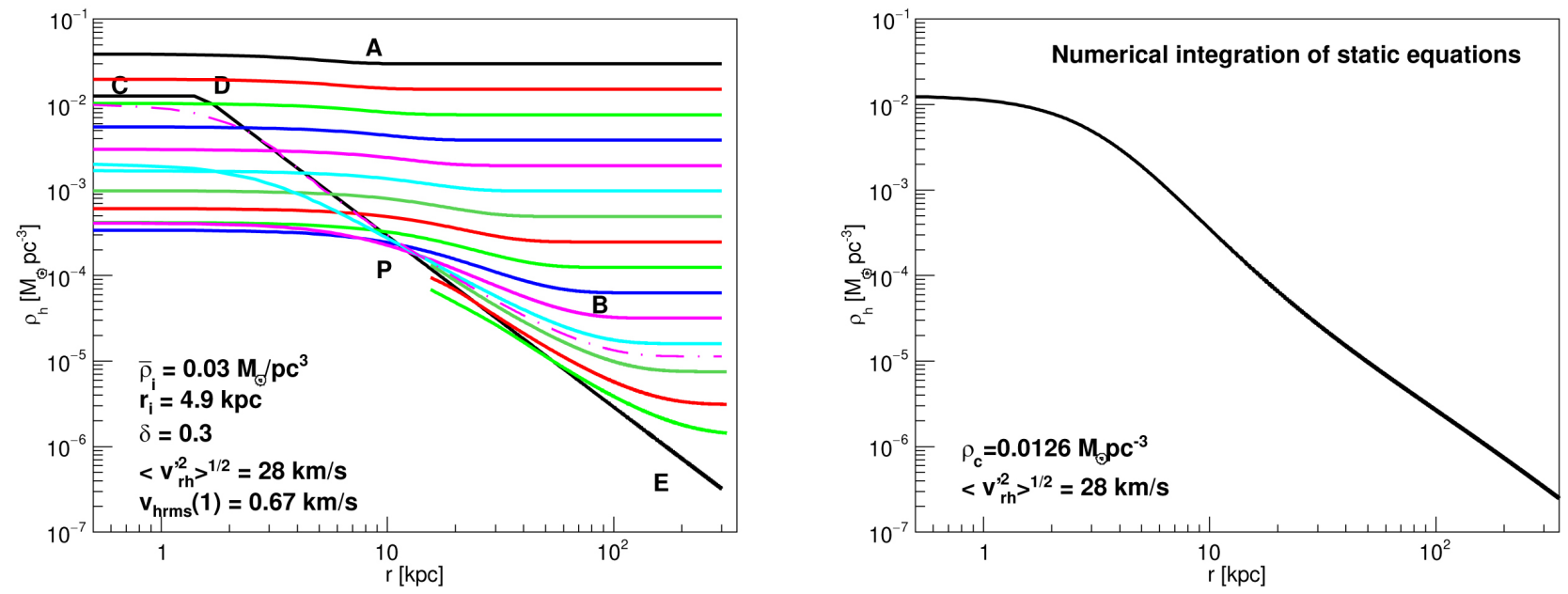

Figure 11. Left: Extended numerical integration of Figure 6 skipping over the pivot point P. Right: Integration of the static equations of Section 2, for comparison with asymptotes C D E. Note that the density run $\rho_{h}(r)$ is determined by the two boundary conditions $\sqrt{\left\langle v_{r h}^{\prime 2}\right\rangle}=28 \mathrm{~km} / \mathrm{s}$, and $\rho_{c}=0.0126 M_{\odot} \mathrm{pc}^{-3}$. 


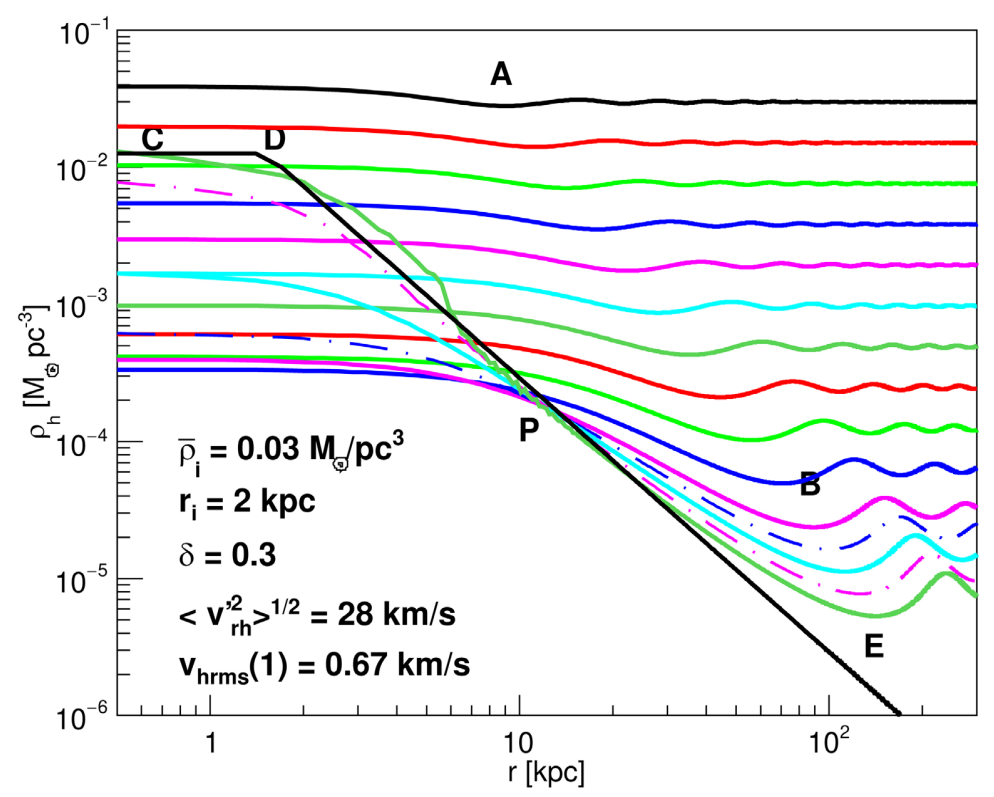

Figure 12. Formation of the dark matter halo of a galaxy with initial density perturbation $\rho_{h}=\bar{\rho}_{i}\left[1+0.3 \cdot \sin \left(r / r_{i}\right) /\left(r / r_{i}\right)\right]$ with $\bar{\rho}_{i}=0.03 M_{\odot} \mathrm{pc}^{-3}$, and $r_{i}=2 \mathrm{kpc}$. The asymptotes of the final dark matter halo $\rho_{h}(r)$, indicated by lines C D E, have $\sqrt{\left\langle v_{r h}^{\prime 2}\right\rangle}=28 \mathrm{~km} / \mathrm{s}$, and $\rho_{c}=0.0126 M_{\odot} / \mathrm{pc}^{3}$.

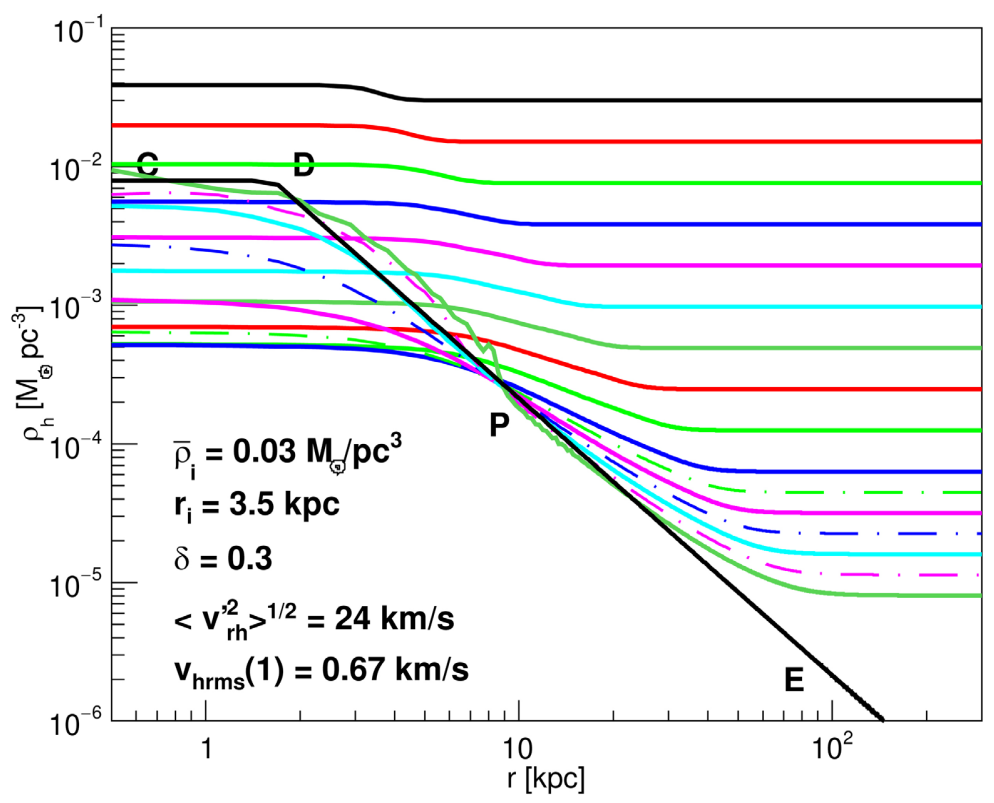

Figure 13. Formation of the dark matter halo of a galaxy with a top-hat initial density perturbation with $\bar{\rho}_{i}=0.03 M_{\odot} \mathrm{pc}^{-3}, \delta_{i}=0.3$, and $r_{i}=3.5 \mathrm{kpc}$. The edges of the step-function have been rounded to allow numerical integration. The asymptotes of the final dark matter halo $\rho_{h}(r)$, indicated by lines C D E, have $\sqrt{\left\langle v_{r h}^{\prime 2}\right\rangle}=24 \mathrm{~km} / \mathrm{s}$, and $\rho_{c}=0.008 M_{\odot} / \mathrm{pc}^{3}$. This figure illustrates that even a top-hat initial perturbation can form a stationary halo adiabatically, due to the feedback of the warm dark matter adiabatic invariant $v_{h \mathrm{rms}}(1)=0.67 \mathrm{~km} / \mathrm{s}$. 
region, corresponding to homogeneous universes, are well known. The adiabatic invariant $v_{h r m s}(1)$ has the same constant value inside and outside of the transition region, and has a cosmological origin in the early universe when perturbations were linear. Note how the core density $\rho_{c}(t) \approx \rho_{c}\left(r_{\min }, t\right)$ decreases, turns around, and increases (as in an overdense homogeneous universe) until the transition region reaches the core halting further increase of $\rho_{c}(t)$. This figure illustrates why we calculate $\sqrt{\left\langle v_{r h}^{2}\right\rangle}$ independent of $r$ using $\rho_{c}\left(r_{\text {min }}, t\right)$, see (16).

Figure 14 illustrates the hierarchical formation of galaxies. Reversing the sign of the perturbation of Figure 12 we can study the expansion of voids, see Figure 15.

To the preceeding examples we may add a velocity perturbation, e.g. $v_{r h}=H r\left(1-\delta_{h} / 3\right)$ for a growing initial mode.

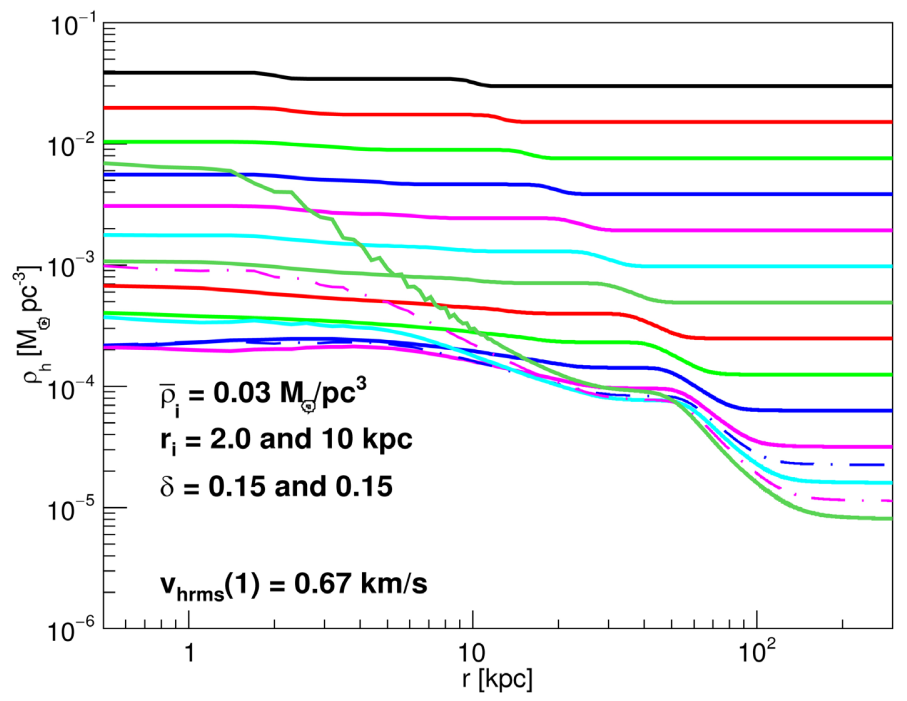

Figure 14. Illustration of the hierarchical formation of galaxies (with two pivot points). The initial density perturbation is a double top-hat.

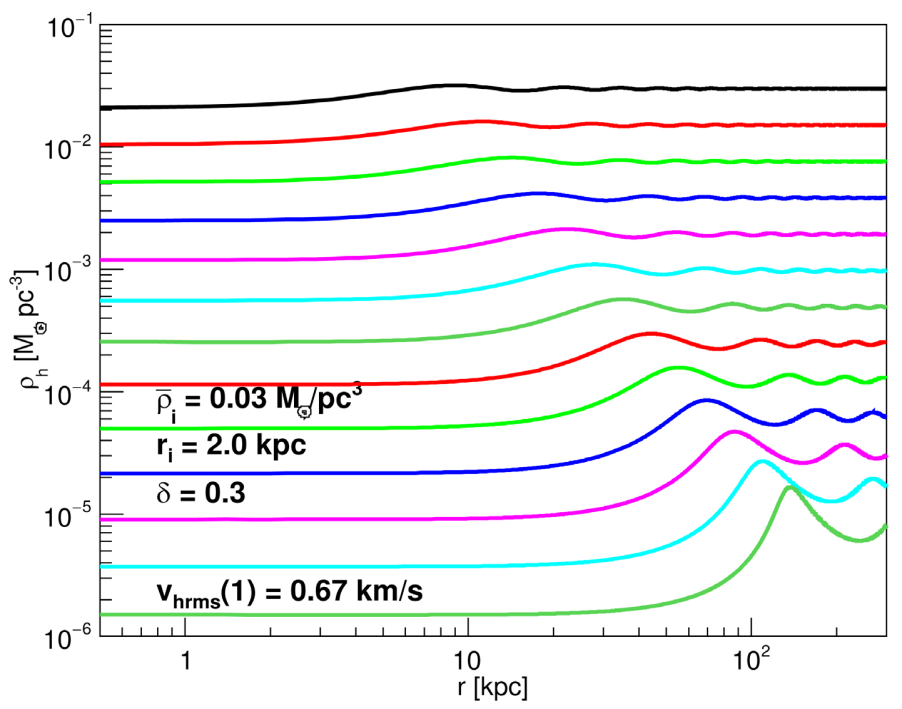

Figure 15. Same as Figure 12, except that the sign of the perturbation has been reversed in order to study the expansion of voids. 


\section{The Adiabatic Invariant $v_{h \mathrm{rms}}(1)$}

Consider a box of volume $V=L^{3}$ with non-relativistic dark matter, and apply periodic boundary conditions: $p_{x}=\hbar k_{x}=\hbar 2 \pi n / L$, with $n=0, \pm 1, \pm 2, \cdots$, and similarly for $p_{y}$ and $p_{z}$. Let $n\left(\mathbf{p}_{n m l}\right)$ be the number of dark matter particles in orbital $\mathrm{nml}$. Then the density, and mean of the velocity squared, are

$$
\rho_{h}=\frac{m_{h}}{V} \sum_{n m l} n\left(\mathbf{p}_{n m l}\right) \propto V^{-1}, \quad\left\langle v_{h}^{2}\right\rangle=\frac{\sum_{n m l}\left|\mathbf{p}_{n m l}\right|^{2} n\left(\mathbf{p}_{n m l}\right)}{m_{h}^{2} \sum_{n m l} n\left(\mathbf{p}_{n m l}\right)} \propto V^{-2 / 3},
$$

where $m_{h}$ is the dark matter particle mass. We note that $\rho_{h} /\left\langle v_{h}^{2}\right\rangle^{3 / 2}$ does not depend on $V$, i.e. it is an adiabatic invariant, so long as the number of dark matter particles per orbital $n\left(\mathbf{p}_{n m l}\right)$ remains constant, as expected for non-interacting particles, or even, on average, for particles with elastic interactions in a relaxed configuration. We will assume that dark matter particles are non-interacting, except for gravity.

Now assume that the box is free-falling, so $V$ varies, and the gas expands or contracts adiabatically conserving $\rho_{h} /\left\langle v_{h}^{2}\right\rangle^{3 / 2}$. A free-falling observer in a density peak in the early universe sees the dark matter gas expand adiabatically, reach maximum expansion, turn around, and contract adiabatically into the core of a galaxy. See, for example, Figure 6. Due to the hierarchical formation of galaxies, galaxies merge and our observer continues seeing adiabatic contractions. See, for example, Figure 14. The end result is that $\rho_{h} /\left\langle v_{h}^{2}\right\rangle^{3 / 2}$ remains constant, as in the early universe, i.e. $\rho_{h} /\left\langle v_{h}^{2}\right\rangle^{3 / 2}$ is of cosmological origin, and we expect $\rho_{h} /\left\langle v_{h}^{2}\right\rangle^{3 / 2}$ to be the same for all free-falling observers at rest with respect to the dark matter, i.e. observers in the cores of relaxed galaxies. And this is indeed what is observed (within statistical and systematic uncertainties), see Figure 16, with far-reaching consequences [2] [4] [13] [17] [18]! It has been argued that the adiabatic invariant might become diluted by "phase space mixing". However, the observations in Figure 16 seem to indicate that, if phase space becomes diluted, then it later becomes un-diluted as the galaxy halo relaxes.

We find it convenient to define the adiabatic invariant in the core of a galaxy as

$$
v_{h \mathrm{rms}}(1) \equiv \sqrt{3}\left\langle v_{r h}^{2}\right\rangle^{1 / 2}\left(\frac{\Omega_{c} \rho_{\text {crit }}}{\rho_{c}}\right)^{1 / 3}
$$

as in (11), where $\left\langle v_{r h}^{2}\right\rangle$ and $\rho_{c}$ are obtained from observed galaxy rotation curves. The mean and standard deviation of 40 well measured galaxies, is

$$
v_{h \mathrm{rms}}(1)=\sqrt{1-\kappa_{h}} \times(0.87 \pm 0.27 \mathrm{~km} / \mathrm{s})
$$

for non-degenerate dark matter, see Figure 4 of [13]. $\kappa_{h}$ is a correction for dark matter halo rotation, estimated to be $\kappa_{h}=0.15_{-0.15}^{+0.35}$ for spiral galaxies [2] [4]. For the interesting cases of fermion or boson dark matter with zero chemical potential in the core of the galaxy, see reference [2]. 


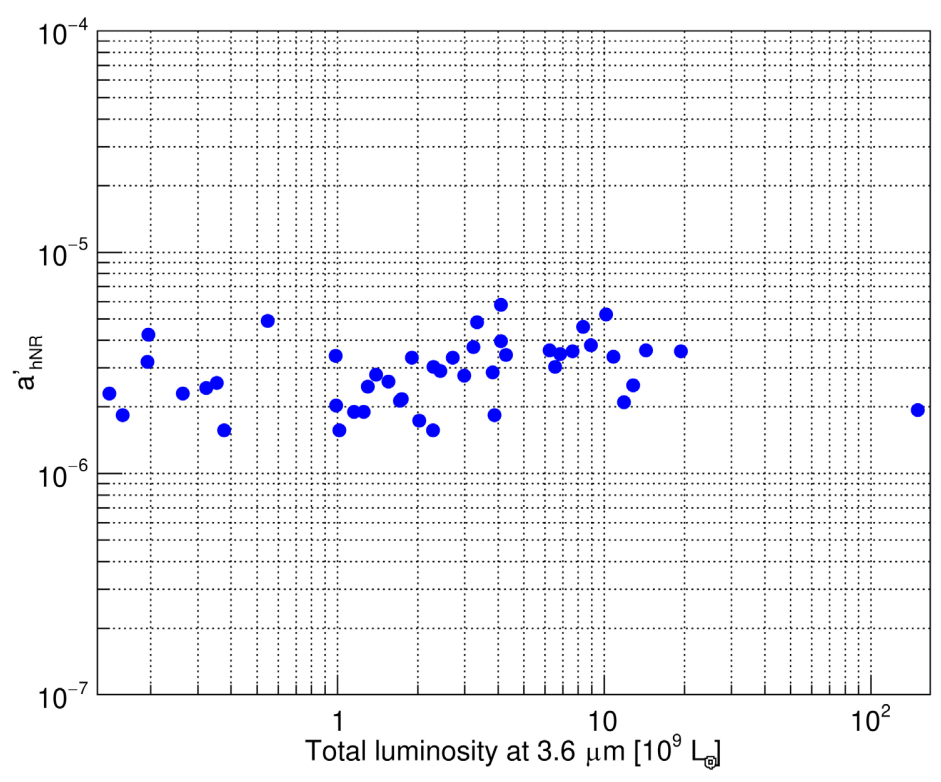

Figure 16. Forty six independent measurements of the expansion parameter $a_{h \mathrm{NR}}^{\prime} \equiv v_{h r \mathrm{rs}}(1) / c$ at which dark matter particles become non-relativistic (uncorrected for dark matter halo rotation). Each measurement was obtained by fitting the rotation curves of a spiral galaxy in the Spitzer Photometry and Accurate Rotation Curves (SPARC) sample [12] with the indicated total luminosity at $3.6 \mu \mathrm{m}$. Figure from [19]. References to the original rotation velocity measurements can be found in [12]. Two of these measurements were given in Figure 4 and Figure 5. Full details of each measurement are presented in [13].

\section{Conclusions}

Let us consider a relaxed galaxy dominated by dark matter. The density run $\rho_{h}(r)$ is determined by just two parameters, i.e. the reduced velocity dispersion $\sqrt{\left\langle v_{r h}^{\prime 2}\right\rangle}$, and the core density $\rho_{c}$. These parameters are obtained from observations of galaxy rotation curves, as explained in Section 2. Our objective, in the present study, is to understand how these two parameters are obtained from the linear density-velocity-gravitational perturbations in the early universe. The halo asymptote $\rho_{h}=\left\langle v_{r h}^{\prime 2}\right\rangle /\left(2 \pi G r^{2}\right)$ is the solution of the isothermal sphere, its radius grows at a velocity $\sqrt{3\left\langle v_{r h}^{\prime 2}\right\rangle}$, and determines the flat rotation velocity $v_{\text {flat }}=\sqrt{2\left\langle v_{r h}^{\prime 2}\right\rangle}$ of test particles. The parameter $\sqrt{\left\langle v_{r h}^{\prime 2}\right\rangle}$ is obtained by numerical integration, starting from the linear perturbation, see Section 3. This asymptotic halo is obtained adiabatically, beyond the pivot point $\mathrm{P}$, even for the top-hat perturbation, see Figure 13.

The second parameter, i.e. the core density $\rho_{c}$, is a mystery in the cold dark matter $\Lambda \mathrm{CDM}$ model. In that model, the core expands, reaches turn-around, collapses, overshoots, followed by splash-back, overshoots again, etc., and several relaxation processes are invoked to reach a virialized state. How can the $o b$ servations in Figure 16 be explained in the cold $\Lambda \mathrm{CDM}$ scenario?

On the other hand, we are able to obtain $\rho_{c}$ if dark matter is warm, for then 
Equations (12), (13), and (14) need to be supplemented by the equation of adiabatic expansion (16) with $v_{h \mathrm{rms}}(1) \neq 0$. In fact, the adiabatic invariant $v_{h \mathrm{rms}}(1)$ not only fixes the central density $\rho_{c}$, but also stabilizes it. The dark matter at $r \approx 0$ expands, reaches turn-around, and begins to collapse. As the core central density $\rho_{c}$ increases adiabatically, its temperature proportional to $\left\langle v_{r h}^{\prime 2}\right\rangle$ also increases, halting the expansion. This negative feedback can be understood by studying Figure 7 and Figure 9. Note that an adiabatic formation of the core is possible, even with no relaxation processes or baryons (at least for an initial linear perturbation sufficiently near Jeans instability, as expected for a power spectrum $P(k) \tau^{2}\left(k / k_{\mathrm{fs}}\right) \propto k^{-n}$, for $k \lesssim k_{\mathrm{fs}}$, with $\left.n \leq 3\right) . v_{r h}$ approaches its equilibrium value $v_{r h}=0$ out to a radius that increases with time with constant velocity $\sqrt{3\left\langle v_{r h}^{\prime 2}\right\rangle}$, populating the tail of the non-relativistic Boltzmann distribution.

The velocity dispersion term in (14) is needed to understand the density run $\rho_{h}(r)$ of relaxed galaxies, see Section 2 . Once this velocity dispersion term is included, Equations (12), (13), and (14) become incomplete since it is then necessary to specify $\left\langle v_{r h}^{2}\right\rangle$, i.e. we need to add the equation of adiabatic expansion (16) for both theoretical and experimental reasons, see Section 4, and Figure 16. The observed dark matter core is evidence that dark matter is warm, see (19).

To understand the baryon density run $\rho_{b}(r)$ we do need to include relaxation processes in the formation, collision, and merger of galaxies, in star formation, in the formation of binaries and black holes, and in radiation of photons and neutrinos. Relaxation allows baryons to settle to the bottom of the dark matter gravitational potential well, and even dominate the central density and gravitational potential with a core, or a cusp of baryons.

If indeed the galaxy central density of dark matter $\rho_{c}$ is determined by the adiabatic invariant $v_{h \mathrm{rms}}(1)$, as supported by the theory described in Section 4, and by the data presented in Figure 16, then $v_{h r m s}(1)$ is of cosmological origin, and provides a detailed, precise, and redundant determination of dark matter properties, see [2] and references therein.

\section{Conflicts of Interest}

The author declares no conflicts of interest regarding the publication of this paper.

\section{References}

[1] Weinberg, S. (2008) Cosmology. Oxford University Press, Oxford.

[2] Hoeneisen, B. (2020) Fermion or Boson Dark Matter? International Journal of Astronomy and Astrophysics, 10, 203-223. https://doi.org/10.4236/ijaa.2020.103011

[3] Zyla, P.A., et al. (Particle Data Group) (2020) The Review of Particle Physics. Progress of Theoretical and Experimental Physics, 2020, Article ID: 083C01.

[4] Hoeneisen, B. (2019) A Study of Dark Matter with Spiral Galaxy Rotation Curves. International Journal of Astronomy and Astrophysics, 9, 71-96. https://doi.org/10.4236/ijaa.2019.92007 
[5] Murphy, J.D., Gebhardt, K. and Adams, J. (2011) Galaxy Kinematics with VIRUS-P: The Dark Matter Halo of M87. The Astrophysical Journal, 729, Article No. 129. https://doi.org/10.1088/0004-637X/729/2/129

[6] Feynman, R.P., Leighton, R.B. and Sands, M. (1963) The Feynman Lectures on Physics. Addison-Wesley Publishing Company, Boston.

[7] Napolitano, N.R., Pota, V., Romanowsky, A.J., Forbes, D.A., Brodie, J.P. and Foster, C. (2014) The SLUGGS Survey: Breaking Degeneracies between Dark Matter, Anisotropy and the IMF Using Globular Cluster Subpopulations in the Giant Elliptical NGC 5846. Monthly Notices of the Royal Astronomical Society, 439, 659-672. https://doi.org/10.1093/mnras/stt2484

[8] Thomas, J., Saglia, R.P., et al. (2005) Regularized Orbit Models Unveiling the Stellar Structure and Dark Matter Halo of the Coma Elliptical NGC 4807. Monthly Notices of the Royal Astronomical Society, 360, 1355-1372.

https://doi.org/10.1111/j.1365-2966.2005.09139.x

[9] Shajib, A.J., Treu, T., Birrer S. and Sonnenfeld A. (2021) Dark Matter Haloes of Massive Elliptical Galaxies at $z \approx 0.2$ Are Well Described by the Navarro-FrenkWhite Profile. Monthly Notices of the Royal Astronomical Society, 503, 2380-2405. https://doi.org/10.1093/mnras/stab536

[10] Wasserman, A., et al. (2018) The SLUGGS Survey: The Inner Dark Matter Density Slope of the Massive Elliptical Galaxy NGC 1407. The Astrophysical Journal, 863, Article No. 130. https://doi.org/10.3847/1538-4357/aad236

[11] Napolitano, N.R., et al. (2010) The PN.S Elliptical Galaxy Survey: A Standard $\Lambda$ CDM Halo around NGC 4374? Monthly Notices of the Royal Astronomical Society, 411, 2035-2053. https://doi.org/10.1111/j.1365-2966.2010.17833.x

[12] Lelli, F., McGaugh, S.S. and Schombert, J.M. (2016) SPARC: Mass Models for 175 Disk Galaxies with Spitzer Photometry and Accurate Rotation Curves. The Astronomical Journal, 152, Article No. 157. https://doi.org/10.3847/0004-6256/152/6/157

[13] Hoeneisen, B. (2019) The Adiabatic Invariant of Dark Matter in Spiral Galaxies. International Journal of Astronomy and Astrophysics, 9, 355-367.

[14] Vogelsberger, M., Marinacci, F., Torrey, P. and Puchwein, E. (2020) Cosmological Simulations of Galaxy Formation. Nature Reviews Physics, 2, 42-66. https://doi.org/10.1038/s42254-019-0127-2

[15] Lapi, A., et al. (2017) Stellar Mass Function of Active and Quiescent Galaxies via the Continuity Equation. The Astrophysical Journal, 847, Article No. 13. https://doi.org/10.3847/1538-4357/aa88c9

[16] Binney, J., and Tremaine, S. (2008) Galactic Dynamics. 2nd Edition, Princeton University Press, Princeton. https://doi.org/10.1515/9781400828722

[17] Hoeneisen, B. (2019) A Study of Dark Matter with Spiral Galaxy Rotation Curves. Part II. International Journal of Astronomy and Astrophysics, 9, 133-141. https://doi.org/10.4236/ijaa.2019.92010

[18] Hoeneisen, B. (2020) Cold or Warm Dark Matter? A Study of Galaxy Stellar Mass Distributions. International Journal of Astronomy and Astrophysics, 10, 57-70. https://doi.org/10.4236/ijaa.2020.102005

[19] Hoeneisen, B. (2021) Adding Dark Matter to the Standard Model. International Journal of Astronomy and Astrophysics, 11, 59-72.

https://doi.org/10.4236/ijaa.2021.111004 